\section{Role of CD36 expression on circulating monocytes subsets in children on regular hemodialysis}

\author{
Noha Mansour ${ }^{1}$, Manal Abdel-Salam ${ }^{1}$, Reham \\ Hammad $^{2}$, Shimaa Moustafa ${ }^{1}$ and Hala Maghraby ${ }^{3}$
}

\author{
${ }^{1}$ Pediatric Department, Faculty of Medicine (for Girls), Al-Azhar \\ University, Egypt. \\ ${ }^{2}$ Clinical Pathology Department, Faculty of Medicine (for Girls), \\ Al-Azhar University, Egypt. \\ ${ }^{3}$ Radiology Department, Faculty of Medicine (for Girls), Al- \\ Azhar University, Egypt.
}

The Egyptian Journal of Immunology Volume 28 (4), 2021: 224-232. www.Ejimmunology.org

\begin{abstract}
Cardiovascular diseases are common in children with chronic kidney disease (CKD). According to studies, monocytes play a role in atherosclerotic vascular disorders. CD36 promotes the binding of oxidised low-density lipoprotein (oxLDL) on monocytes, however its role in atherosclerosis is unclear. We aimed to assess the frequency of monocyte subsets in CKD children, and to determine CD36 differential expression on monocyte subsets and its association with the risk of atherosclerotic incidents in those patients. This case-control study included 40 children with CKD and 40 apparently healthy children as controls. We investigated the frequency of total monocyte and monocyte subsets using CD14/CD16. Also, we assessed CD36 differential expression on circulating subsets using flow cytometry. In addition to Doppler ultrasound assessment of the intimal medial thickness (IMT) and peak systolic velocity (PSV) of the main arteries, with routine laboratory investigations of both groups. There was a significant increase in median values of total circulating monocyte percentage in patients than controls $(P=0.001)$. Also, there was a significant increase in the percentage of classical monocyte (CD14high/CD16-) and non-classical monocytes subset (CD14low/CD16+) in patients when compared to controls $(P=0.027)$ and $(P=0.001)$, respectively. There was a significant decrease in the median values of CD36 Mean fluorescence intensity (MFI) expressed on classical, non-classical and intermediate monocytes in patients than the control group $(P=0.001),(P=0.001)$ and $(P=0.002)$, respectively. CD36 MFI expressed on classical, non-classical and intermediate monocytes negatively correlated with cholesterol, triglyceride, systolic, diastolic blood pressure and the IMT of the femoral artery. In conclusion, the increase in the frequency of monocytes, particularly the classical and nonclassical subsets, may be a key component in atherosclerosis pathogenesis in children on hemodialysis. Low CD36 expression on monocyte subsets may be involved in the pathogenesis of atherosclerosis in children on hemodialysis.
\end{abstract}

Keywords: Atherosclerosis, Chronic kidney disease, CD36, Hyperlipidemia, Monocyte subsets. 


\section{Introduction}

Patients with chronic kidney disease (CKD) are at high risk of premature mortality, mainly from cardiovascular causes. The association between CKD on hemodialysis and accelerated atherosclerosis was described more than 40 years ago. However, it has been suggested that the increase in the atherosclerosis risk was noted in early CKD stages. ${ }^{1-2}$

There is no specific treatment for factors predisposing atherosclerosis in CKD. A role for circulating monocytes is claimed, with persistent inflammation due to accumulation of urea, and defect in cytokine excretion..$^{3-5}$ The responses of blood vessels to circulating monocytes are involved in endothelial dysfunction reported in CKD, occurrence of calcification, and atherogenesis. ${ }^{6-7}$

Monocytes are short-lived innate immune cells. $^{8-9}$ Studies highlighted the role that monocyte play in the development of atherosclerosis, as monocyte differentiation is shifted into lipid-laden macrophages, contributing to the progression of atherosclerotic lesions. ${ }^{10-11}$

Monocytes are divided into three subsets based on CD14 and CD16 expression: classical (CD14high/CD16-), intermediate (CD14high/ CD16+), and non-classical (CD14low/ CD16+), of which their expression of surface antigen CD16 can distinguish 10- 20\%. Many studies have reported increased numbers of $\mathrm{CD} 16+$ monocytes in CKD and linked that to development of cardiovascular complications. ${ }^{12-}$ 13 The link between CD16+ monocytes and accelerated atherosclerosis is due to the ability of this type of monocytes for lipoprotein scavenging and cholesterol efflux. ${ }^{14-15}$

The CD36 is a membrane glycoprotein that is expressed on monocytes and macrophages. CD36 is claimed to play a role in formation of atherosclerotic lesions by promoting endocytosis of oxidized low-density lipoprotein (oxLDL) and forming foam cells, which is the initial stage of atherosclerotic lesions. ${ }^{16-17}$ Also, binding of oxLDL via CD36 inhibits macrophage migration, which is a macrophage-trapping mechanism. ${ }^{18}$
In the current study we aimed to assess the total monocyte percentage and its subsets using CD14 and CD16. In addition, we intended to determine CD36 differential expression on monocyte subsets and its association with the risk of atherosclerotic incidents in children undergoing hemodialysis.

\section{Subjects and Methods}

\section{Subjects}

This case-control study included 40 pediatric patients who fulfilled the criteria for definition and classification of patients with chronic kidney diseases. ${ }^{19}$ They were on regular hemodialysis for 4 hours/setting, three times weekly, with low flux polysulphone dialyzer 4008 Fresenius machines. Their ages ranged from 2 to 18 years. A group of 40 apparently healthy, age and sex matched children served as controls. Exclusion criteria included children with primary vascular disorder, primary immune deficiency, acute or chronic infection and CKD children on immunosuppression or antibiotics therapy.

Participants were subjected to detailed history taking, and laboratory investigations. The study protocol was reviewed and approved by the Research Ethical Committee of the Faculty of Medicine for Girls Institutional Research Board, Al-Azhar University (approval date; February 2020). Informed consents from the children parents were taken. This study was conducted at the Pediatric (nephrology and hemodialysis), Clinical Pathology, and Radiology departments, Al-Azhar University.

\section{Sampling and methodology}

Sample collection and laboratory investigations of children pre-HD

From each participant, $6 \mathrm{ml}$ of blood was withdrawn after $12 \mathrm{hrs}$ of fasting. Samples were divided into two portions; the first $2 \mathrm{ml}$ of blood was added to an EDTA tube for assessing complete blood count using an automated hematology analyzer (Sysmex XK-21, Japan), and for ESR and flow cytometry assay. The other $4 \mathrm{ml}$ samples were added to plan tubes for serum separation. Then serum was divided to 
two aliquots. The first aliquot was used for measuring urea, creatinine, lipid profile, total calcium (Ca), and phosphorus (Ph) using an automated blood chemistry analyzer (Cobas Integra 400 plus, Roche diagnostics, Germany). The second aliquot was for measuring sodium $(\mathrm{Na}+)$ and potassium $(\mathrm{K}+)$, detected by an electrolyte analyzer (AVL, 9180 Roche diagnostics, Germany).

Flow cytometry assay: An aliquot of $50 \mu \mathrm{l}$ of fresh blood was incubated with $5 \mathrm{ul}$ of the following: CD14- PE-conjugated Ab (Immunotech; Beckman Coulter, Marseille, France, cat. no. A07764, lot. no.25), CD16-FITCconjugated Ab (Immunotech; Beckman Coulter, Marseille, France; cat. no. P59232AA, lot no. 200105). CD14 and CD16 were used for monocytes subsets detection. CD36 APCconjugated Abs (Elabscience. Cat no, E-ABF1164. Lot no, FW1227, China) were incubated for $10 \mathrm{~min}$. RBCs were lysed. The sample was then washed using phosphate buffer saline before being transferred to the flow cytometry device (BD, Biosciences, San Jose, California, USA). Isotype controls were used to set cut-offs.

The initial gate was taken on FS/SS dot plot on the monocytes area (R1). From the total monocytes population (R1), a quadrant plot was taken showing CD14 on Y-axis and CD16 on Xaxis. According to their expression; the monocyte's three subsets were identified to; classical monocytes CD14 high CD16-(R2), nonclassical CD14 low CD16 high (R3) and intermediate monocytes CD14 high CD16+ (R4). A single histogram was drawn on each subset to detect CD36 Mean fluorescence intensity (MFI) on a positive population under the M1 marker. Flow-cytometry was conducted in the Allergy and Immunology Center, AL-Azhar University, on multicolor FACS Calibur (BD, Biosciences, San Jose, California, USA), for data analysis. The gating strategy is illustrated in (Figure, 1).

\section{Radiological investigations}

Intimal medial thickness (IMT) and Peak systolic velocity (PSV) were measured using an ultrasound system, the 2-Doppler Ultrasound
Machine (Mylab 50 X Vision, Esaote. Genoa, Florence, Italy) apparatus in Al-Zahraa University hospital. The IMT is the distance between the leading edge of the lumen-intima interface and the media-adventitia interface.

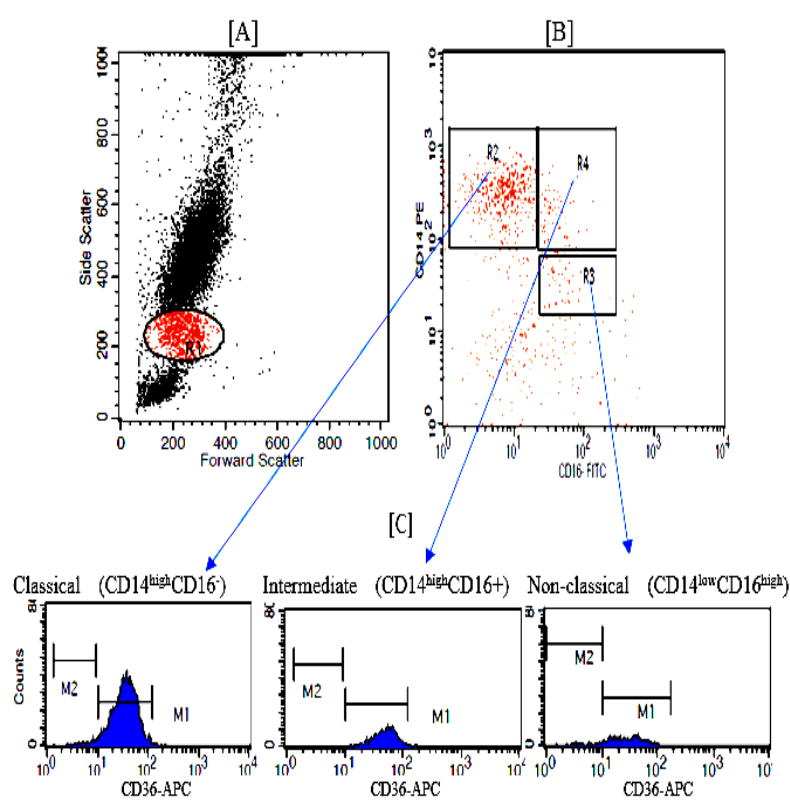

Figure 1. Gating strategy for detection of CD36MFI on different monocytes subsets.

\section{Statistical analysis}

Statistical Package for Social Science (IBM SPSS) version 23 was used for data collection, coding, and data entry. Comparisons between quantitative variables were done using the Mann-Whitney tests for comparing categorical data. Spearman correlation coefficients was used to assess the correlation between two studied parameters in the same group. A $P$ value less than 0.05 (typically $\leq 0.05$ ) was considered statistically significant.

\section{Results}

Table 1 shows a significant increase in blood pressure (systolic \& diastolic). Also, there was a significant increase in serum $K, C R P, E S R$ in patients than in the control group. Meanwhile, there was a significant decrease in counts of RBCs, WBCs, platelets, level of $\mathrm{Hb}$ and serum $\mathrm{Ca}$ in patient's than in the control group. 
Table 1. Comparison between the control group and patients' group regarding age, sex, blood pressure, and routine laboratory data.

\begin{tabular}{|c|c|c|c|}
\hline Variable & $\begin{array}{l}\text { Control group } \\
(\text { No. }=40) \\
\text { Mean } \pm \text { SD }\end{array}$ & $\begin{array}{l}\text { Patients group } \\
(\text { No. }=40) \\
\text { Mean } \pm \text { SD }\end{array}$ & ${ }^{*} P$-value \\
\hline Age (yrs) & $10.075 \pm 4.097$ & $9.875 \pm 3.864$ & NS \\
\hline Male & 15 (37.5\%) & 15 (37.5\%) & \multirow{2}{*}{ NS } \\
\hline Female & $25(62.5 \%)$ & $25(62.5 \%)$ & \\
\hline Systolic (mmHg) & $105.38 \pm 3.40$ & $122.33 \pm 7.74$ & 0.001 \\
\hline Diastolic (mmHg) & $68.4 \pm 1.69$ & $86.35 \pm 7.62$ & 0.003 \\
\hline $\operatorname{TLC} \times 10^{3} / \mu \mathrm{l}$ & $6.40 \pm 0.78$ & $9.32 \pm 2.25$ & 0.004 \\
\hline $\mathrm{RBC} s \times 10^{6} / \mu \mathrm{l}$ & $4.97 \pm 0.39$ & $3.09 \pm 0.59$ & 0.001 \\
\hline $\mathrm{Hb}(\mathrm{gm} / \mathrm{dL})$ & $12.06 \pm 0.98$ & $9.36 \pm 1.17$ & 0.01 \\
\hline PLT $\times 10^{3} / \mu \mathrm{l}$ & $291.13 \pm 38.13$ & $234.43 \pm 35.32$ & 0.01 \\
\hline ESR / hour & $16.63 \pm 4.36$ & $36.4 \pm 13.32$ & 0.01 \\
\hline $\mathrm{CRP}(\mathrm{mg} / \mathrm{dL})$ & $4.40 \pm 1.37$ & $16.43 \pm 5.07$ & 0.01 \\
\hline $\mathrm{K}(\mathrm{meq} / \mathrm{L})$ & $4.03 \pm 0.3$ & $6.24 \pm 0.7$ & 0.001 \\
\hline $\mathrm{Ca}(\mathrm{mg} / \mathrm{dL})$ & $9.88 \pm 0.44$ & $8.77 \pm 0.82$ & 0.01 \\
\hline $\mathrm{CHO}(\mathrm{mg} / \mathrm{dL})$ & $106.72 \pm 1.98$ & $169.64 \pm 18.14$ & 0.01 \\
\hline$\overline{T G}(\mathrm{mg} / \mathrm{dL})$ & $84.67 \pm 7.24$ & $171.9 \pm 24.67$ & 0.001 \\
\hline
\end{tabular}

TLC, total leucocyte count; $\mathrm{Hb}$, Hemoglobin; PLT; platelet; TG, triglyceride; CRP; C reactive protein; $\mathrm{CHO}$, cholesterol; $\mathrm{Ca}$, calcium; Ph, phosphorus; $\mathrm{Na+}$, sodium; $\mathrm{K}+$, potassium. $P>0.05$ is not significant (NS). *Independent t-test.

Table 2 shows a significant increase in the median level of total monocyte \%, classical monocytes \% (CD14 high/CD16-) and nonclassical monocytes \% (CD14low/ CD16+) in the patient's group compared to the controls (Figure, 2). However, there was no significant difference in intermediate monocytes \% (CD14high/CD16+) in the patient's group compared to the controls. Meanwhile, there was a significant decrease in CD36 MFI on (classical, nonclassical, and intermediate) monocytes in the patient's than in the control group (Figure, 3 ).

As shown in Table 3, there was a significant increase in the IMT of the femoral, brachial, radial, and ulnar arteries, with a significant decrease in their peak systolic velocity of carotid, brachial, and ulnar arteries in the CKD patient's than in the control group. The correlation of CD36 expression on the monocyte's three subsets with other study parameters is shown in Table 4.

Table 2. Comparison between the patients and the control groups regarding total monocytes \%, monocyte's three subsets \% and their CD36 MFI expression.

\begin{tabular}{lccc}
\hline Variable & $\begin{array}{c}\text { Control group No.40 } \\
\text { Median (IQR) }\end{array}$ & $\begin{array}{c}\text { Patients group No.40 } \\
\text { Median (IQR) }\end{array}$ & *P-value \\
\hline $\begin{array}{l}\text { Total monocytes \% } \\
\text { Classical monocytes \% } \\
\text { (CD14high/CD16-) }\end{array}$ & $3.27(2.8-4.55)$ & $5.88(4.05-7.47)$ & 0.001 \\
\hline $\begin{array}{l}\text { Non-classical monocytes \% } \\
\text { (CD14low/ CD16+) }\end{array}$ & $2.26(1.71-3.29)$ & $3.33(1.75-4.63)$ & 0.027 \\
\hline $\begin{array}{l}\text { Intermediate monocytes \% } \\
\text { (CD14high/CD16+) }\end{array}$ & $0.14(0.09-0.18)$ & $0.32(0.18-0.53)$ & 0.001 \\
\hline CD36 MFI on Classical monocytes & $0.28(0.16-0.49)$ & $0.31(0.11-0.46)$ & NS \\
\hline CD36 MFI on non-classical monocytes & $23.09(19.24-48.05)$ & $17.2(14.9-27.4)$ & 0.001 \\
\hline $\begin{array}{l}\text { CD36 MFI on Intermediate monocytes } \\
\text { MFI, Mean fluorescence intensity } P>0.05 \text { is not significant (NS). }{ }^{*} \text { Mann-Whitney test. }\end{array}$ & & 0.002 \\
\hline
\end{tabular}



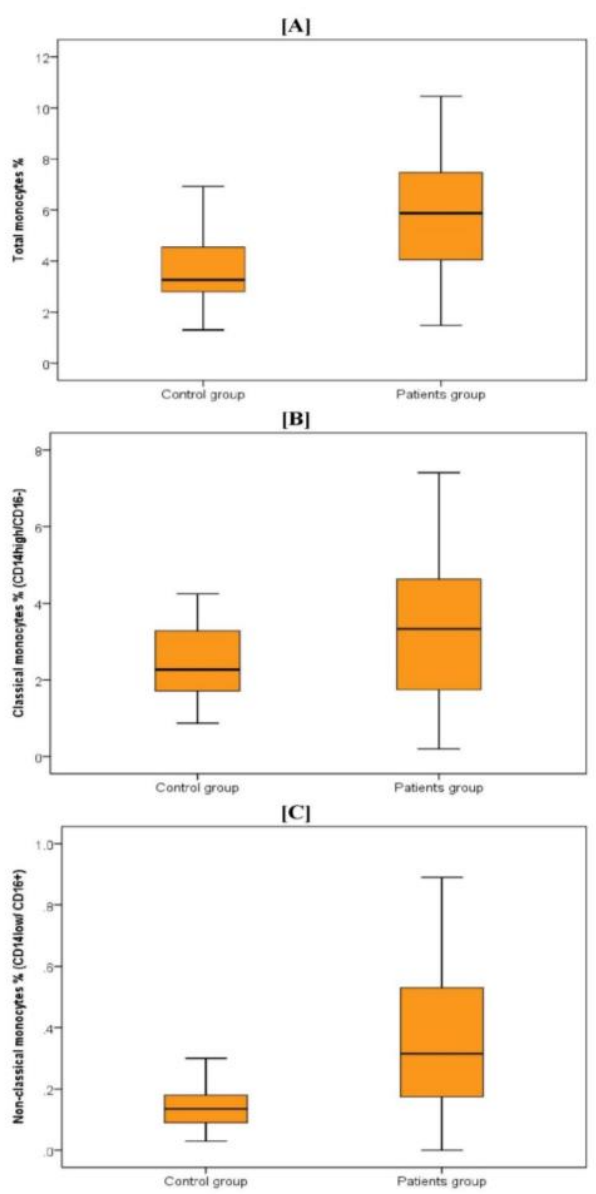

Figure 2. Comparison of total, classical and non-classical monocytes in the studied groups. Children with CKD shows significant increase in percentage of $[A]$ total monocytes $(P=0.000),[\mathrm{B}]$ Classical monocytes $(P=0.027)$, and $[\mathrm{C}]$ Non-classical monocytes $(P=0.000)$ when compared to the control group.
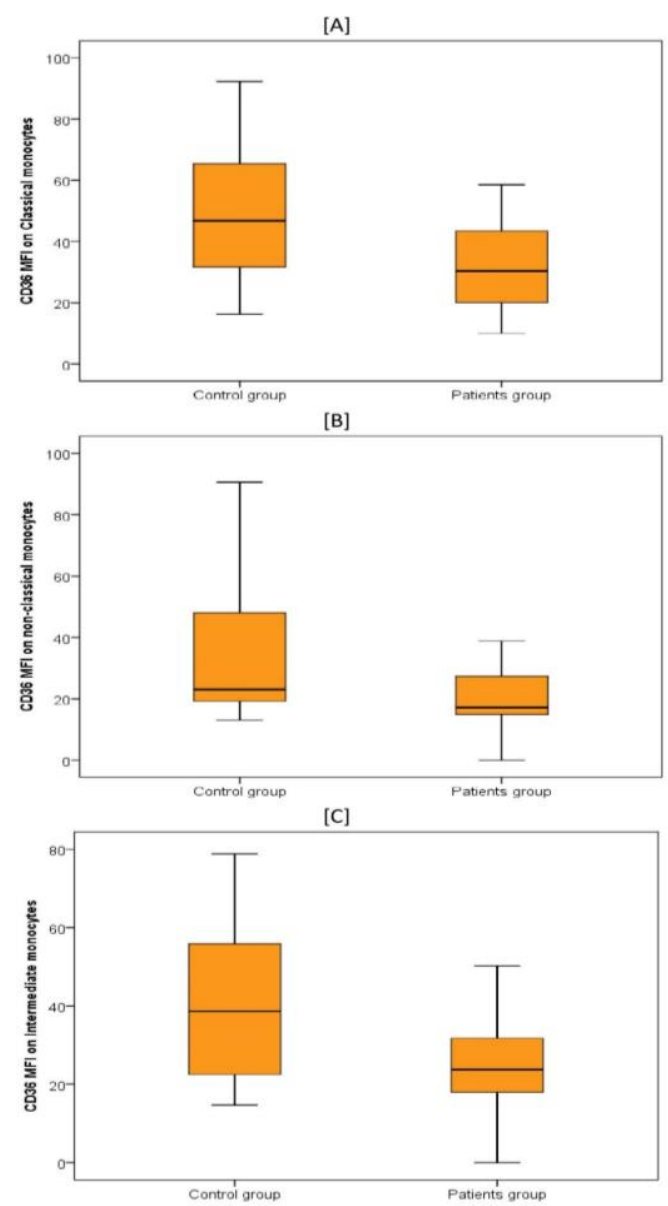

Figure 3. Comparison of CD36 MFI on monocyte's three subsets in the studied groups. Children with CKD shows a significant decrease in CD36 MFI in children with CKD when compared to the control group regarding: [A] Classical monocytes $(P=0.000),[B]$ Non-classical monocytes $(P=0.001)$, and $[C]$ Intermediate monocytes $(P=0.002)$.

Table 3. Comparison between the patients and the control groups regarding intima-media thickness (IMT) and peak systolic velocity (PSV) of the main arteries.

\begin{tabular}{lccc}
\hline & $\begin{array}{c}\text { Control group(No. }=40) \\
\text { Mean } \pm \text { SD }\end{array}$ & $\begin{array}{c}\text { Patients group(No. }=40) \\
\text { Mean } \pm \text { SD }\end{array}$ & *P-value \\
\hline IMT (mm) & & & \\
Carotid & $0.32 \pm 0.036$ & $0.51 \pm 0.01$ & 0.001 \\
Brachial & $0.046 \pm 0.01$ & $0.057 \pm 0.01$ & 0.001 \\
Ulnar & $0.043 \pm 0.01$ & $0.05 \pm 0.01$ & 0.001 \\
Radial & $0.042 \pm 0.01$ & $0.05 \pm 0.01$ & 0.001 \\
Femoral & $0.055 \pm 0.01$ & $0.069 . \pm 0.01$ & 0.001 \\
\hline PSV (mm) & & & \\
Carotid & $52.8 \pm 8.16$ & $47.35 \pm 4.81$ & 0.002 \\
Brachial & $55.65 \pm 8.14$ & $46.53 \pm 5.56$ & 0.001 \\
Ulnar & $43.53 \pm 6.26$ & $38.8 \pm 6.36$ & 0.001 \\
Radial & $40.75 \pm 7.42$ & $39.68 \pm 6.59$ & $\mathrm{NS}$ \\
Femoral & $60 \pm 8.54$ & $57.43 \pm 9.88$ & $\mathrm{NS}$ \\
\hline
\end{tabular}

IMT, intima-media thickness; PSV, peak systolic velocity. $P>0.05$ is not significant (NS). *Independent t-test. 
Table 4. Correlation between MFI of CD36 expression on monocyte's three subsets and the study parameters

\begin{tabular}{|c|c|c|c|c|c|c|}
\hline \multirow[t]{2}{*}{ Variable } & \multicolumn{2}{|c|}{$\begin{array}{l}\text { CD36 MFI on } \\
\text { classical monocyte }\end{array}$} & \multicolumn{2}{|c|}{$\begin{array}{l}\text { CD36 MFI on } \\
\text { non classical monocyte }\end{array}$} & \multicolumn{2}{|c|}{$\begin{array}{l}\text { CD36 MFI on } \\
\text { intermediate } \\
\text { monocyte }\end{array}$} \\
\hline & $r$ & $P$-value & $r$ & $p$-value & $r$ & $P$-value \\
\hline $\mathrm{BMI}$ & 0.052 & NS & -0.215 & 0.055 & -0.015 & NS \\
\hline $\mathrm{TLC} / \mathrm{m}^{3}$ & 0.343 & 0.002 & 0.158 & 0.162 & 0.365 & 0.001 \\
\hline $\mathrm{PLT} / \mathrm{m}^{3}$ & 0.184 & NS & 0.150 & 0.183 & 0.133 & NS \\
\hline$\overline{\mathrm{CRP}}$ & -0.407 & 0.000 & -0.347 & 0.002 & -0.329 & 0.003 \\
\hline ESR / hour & -0.272 & 0.015 & -0.298 & 0.007 & -0.222 & 0.048 \\
\hline Urea (mg/dL) & -.0388 & 0.000 & -0.368 & 0.001 & -0.358 & 0.001 \\
\hline Creatinine $(\mathrm{mg} / \mathrm{dL})$ & -0.386 & 0.000 & -0.338 & 0.002 & -0.338 & 0.002 \\
\hline $\mathrm{Ca}(\mathrm{mg} / \mathrm{dL})$ & 0.241 & 0.031 & 0.144 & 0.202 & 0.170 & NS \\
\hline $\mathrm{Ph}(\mathrm{mg} / \mathrm{dL})$ & -0.379 & 0.001 & -0.299 & 0.007 & -0.295 & 0.008 \\
\hline $\mathrm{Na}(\mathrm{meq} / \mathrm{L})$ & -0.377 & 0.001 & -0.307 & 0.006 & -0.303 & 0.006 \\
\hline $\mathrm{K}$ (meq/L) & -0.357 & 0.001 & -0.320 & 0.004 & -0.327 & 0.003 \\
\hline $\mathrm{CHO}(\mathrm{mg} / \mathrm{dL})$ & -0.323 & 0.003 & -0.311 & 0.005 & -0.269 & 0.016 \\
\hline $\mathrm{TG}(\mathrm{mg} / \mathrm{dL})$ & -0.424 & 0.000 & -0.326 & 0.003 & -0.395 & 0.001 \\
\hline Diastolic (mmHg) & -0.415 & 0.000 & -0.315 & 0.004 & -0.391 & 0.001 \\
\hline Systolic (mmHg) & -0.320 & 0.004 & -0.321 & 0.004 & -0.286 & 0.010 \\
\hline \multicolumn{7}{|l|}{ IMT (mm) } \\
\hline Carotid & -0.146 & NS & -0.114 & 0.313 & -0.107 & NS \\
\hline Brachial & -0.032 & NS & -0.115 & 0.310 & -0.033 & NS \\
\hline Ulnar & -0.214 & 0.056 NS & -0.172 & 0.128 & -0.155 & NS \\
\hline Radial & -0.169 & NS & -0.276 & 0.013 & -0.169 & NS \\
\hline Femoral & -0.319 & 0.004 & -0.385 & 0.000 & -0.289 & 0.009 \\
\hline \multicolumn{7}{|l|}{$\mathrm{PSV}(\mathrm{mm})$} \\
\hline Carotid & 0.182 & NS & 0.246 & 0.028 & 0.124 & NS \\
\hline Brachial & 0.229 & 0.041 & 0.307 & 0.006 & 0.162 & NS \\
\hline Ulnar & 0.089 & NS & 0.137 & 0.225 & 0.067 & NS \\
\hline Radial & 0.094 & NS & -0.017 & 0.878 & 0.010 & NS \\
\hline Femoral & 0.111 & NS & 0.086 & 0.449 & 0.078 & NS \\
\hline
\end{tabular}

TLC, total leucocyte count; Hb, Hemoglobin; PLT; platelet; TG, triglyceride; CRP; C reactive protein; $\mathrm{CHO}$, cholesterol; $\mathrm{Ca}$, calcium; Ph, phosphorus; Na+, sodium; K+, potassium; IMT, intima-media thickness; PSV, peak systolic velocity.

$P>0.05$ is not significant (NS).

\section{Discussion}

Several variables contribute to the accelerated atherogenic process in CKD patients. In this study, we used flow cytometry to assess the involvement of monocyte cells and their three subsets, as well as CD36 expression on these subsets, as a nontraditional actor in the acceleration of atherosclerosis and cardiovascular risk. The median values of total circulating monocyte percentage in patients were significantly higher than in controls $(P=0.001)$. Also, there was a significant increase in the percentage of classical monocyte
(CD14high/CD16-) and non-classical monocytes subset (CD14low/CD16+) in patients when compared to controls $(P=0.027)$ and $(P=0.001)$, respectively.

Previous publications linked between monocyte aberrations and CKD patients. Uremia has been linked to an increase in the CD16+ monocyte subgroup. CD16+ monocytes are smaller, inflammatory, and adherent. Hemodialysis can exacerbate monocyte abnormalities. ${ }^{7}$ Inflammation is caused by uremic toxins. Classic monocytes secrete a considerable number of pro-inflammatory 
substances during the inflammatory phase. Nonclassical monocytes are always patrolling the vascular endothelium and can clear the vasculature of debris and dead cells. ${ }^{8}$

The present study was in line with a study by Carmona et al., 2020 which reported a significant increase in non-classical monocytes in CKD patients compared to controls. ${ }^{20}$ Also, CKD patients on hemodialysis exhibit increased CD14low/CD16+ monocytes, according to a previous study by SahBandar et al., 2019. ${ }^{21}$ Similarly, Rogacev et al., 2011 found that CKD patients had increased CD16+ monocyte. ${ }^{22}$ Chiu et al., 2018 stated that CD14high CD16+ monocytes as well as the CD14lowCD16+ monocytes increase in quantity with age and are even higher in end stage renal disease (ESRD) patients. ${ }^{23}$ The state of chronic inflammation associated with ESRD was responsible for the rise in CD16 expression on monocytes. ${ }^{2}$

The CD36 is a multi-functional scavenger receptor of the class $B$ family that regulates lipid metabolism and immunological responses. ${ }^{8}$ In the current study, there was a significant decrease in the median values of CD36 MFI expressed on classical, non-classical and intermediate monocytes in patient's group than in the control group $(P=0.001),(P=0.001)$ and $(P=0.002)$, respectively. A previous study reported that CD36 serves as a signaling hub protein at the crossroads of inflammation, lipid metabolism, and fatty acid metabolism and that patients with atherosclerosis had high levels of serum soluble CD36. ${ }^{24}$ This could clarify the reason behind the decrease in the attached cellular form of CD36, observed in the current study.

The CD36 MFI expressed on classical, nonclassical and intermediate monocytes negatively correlated with cholesterol, triglyceride, systolic and diastolic blood pressure indicating that CD36 probably plays a role in the development of atherosclerosis. Choromańska et al., 2017 reported that CD36 has independent ability through its functions as a scavenger receptor to bind to three major classes of ligands [modified phospholipids, long chain fatty acids (LCFA) and proteins containing structural domains of thrombospondin homologs]. CD36 facilitates translocation of LCFA in adipocytes, hepatocytes, and heart and skeletal myocytes, where LCFA are significant energy-producing substrates. ${ }^{25}$ Also, Glatz, and Luiken., 2018 stated that CD36 has been linked to dysregulated fatty acid and lipid metabolism in pathophysiological conditions. ${ }^{26}$

In the current study, CD36 MFI on all monocyte's subsets showed a negative correlation with femoral IMT. Such observation, regarding CD36 expression on monocytes, may suggest that these cells play a protective role in preventing atherosclerosis progression in CKD patients. This can be attributed to reduced CD36 expression on circulating monocytes in the present study, accordingly, its role in lipid translocation is compromised, as previously reported. ${ }^{25}$

Our findings disagree with those indicating that CD36 promoting endocytosis of oxLDL in macrophages, forming foam cells, which is the initial stage of atherosclerotic lesions, ${ }^{16-17}$ and that binding of oxLDL via CD36 inhibits macrophage migration, paving the way for atherosclerosis pathogenesis ${ }^{8}$

Lim et al., 2021, highlighted the importance of CD36 in regulating critical physiological processes and cellular events in the initial and progression phases of atherosclerosis, which occur in CKD. ${ }^{27}$ Another study by Liakopoulos et al., 2018, evaluated monocyte expression of CD36 in CKD patients on hemodialysis and revealed a statistically significant two-fold increase in CD36 expression on monocytes in hemodialysis patients than in control. ${ }^{28}$ Also, in disagreement with our study findings, Girndt et al., 2020, reported a significant increase in CD36 expressed on white blood cells in CKD patients. ${ }^{29}$ Moreover, Biswas et al., 2021, reported that CD36 is increased in hyperlipidemic mice. ${ }^{30}$

Such discrepancy between our study findings and these reported by others could be attributed to difference of methodologies used in detecting CD36 expression, as we used flow cytometry to assess surface expression on cellularly attached CD36 on monocytes. However, we did not measure detached soluble CD36, part of the extracellular segment of CD36 cleaved by a plasma protease as reported by 
Tian et al., $2020^{24}$. Finally, the role of CD36 in atherosclerosis is complex and conflicting. It seems that both CD36 overexpression and deficiency predispose to metabolic complications.

In conclusion, our findings suggest that the increases in the frequency of circulating monocytes, mainly the classical and nonclassical subsets may be a critical factor in initiating atherosclerosis pathogenesis in children on hemodialysis. Low CD36 expression on monocyte subsets may have a role in the development of atherosclerosis in hemodialysis patients.

\section{Author Contributions}

NM; collection of samples and ethical approvals, MA; paper proposal, analysis of statistical data, discussion writing and revision, $\mathrm{RH}$; sampling and practical aspect of investigating monocytes subsets and CD36 expression by Flow cytometry, analysis of its data, revision and correspondence of the paper, SM; responsible for clinical data and shared in discussion writing, HM; radiological work

\section{Declaration of Conflicting Interests}

The author(s) declared no potential conflicts of interest with respect to the research, authorship, and/or publication of this article.

\section{Funding}

The author(s) denies receipt of any financial support for the research, authorship, and/or publication of this article.

\section{Ethical approval}

The study protocol was reviewed and approved by the Research Ethical Committee of the Faculty of Medicine for Girls Institutional Research Board, AlAzhar University (approval date; February 2020).

\section{Informed consent}

Informed consents from the children parents were taken.

\section{ORCID iD}

Reham Hammad iD https://orcid.org/0000-00028967-9106

\section{References}

1. Valdivielso JM, Rodríguez-Puyol D, Pascual J, et al. (2019). Atherosclerosis in chronic kidney disease: more, less, or just different? Arteriosclerosis, thrombosis, and vascular biology, 39(10), 19381966.

2. Praveen G (2020). Correlation of Carotid Artery Tunica Intima Medial Thickness in Different Stages of Chronic Kidney Disease (CKD) as a Marker of Atherosclerosis (Doctoral dissertation, Tirunelveli Medical College, Tirunelveli).

3. Tunbridge MJ, and Jardine AG. (2019). Cardiovascular complications of chronic kidney disease. Medicine, 47(9), 585-590.

4. Silva EH, Wickramatilake CM, Lekamwasam S, et al. (2019). Vascular dysfunction and atherosclerosis in chronic kidney disease; A distinct entity. Journal of nephropathology, 8 (2).

5. Sampath $P$, Moideen K, Ranganathan UD and Bethunaickan R. (2018). Monocyte subsets: phenotypes and function in tuberculosis infection. Frontiers in immunology, 9, 1726.

6. Gregg LP, Tio MC, Li X, et al. (2018). Association of monocyte chemoattractant protein-1 with death and atherosclerotic events in chronic kidney disease. American journal of nephrology, 47(6), 395-405.

7. Bowe B. Xie Y., Xian H, Li T, et al. (2017). Association between monocyte count and risk of incident CKD and progression to ESRD. Clinical Journal of the American Society of Nephrology, 12(4), 603-613.

8. Zhao Y, Zou W, Du J, et al. (2018). The origins and homeostasis of monocytes and tissue-resident macrophages in physiological situation. J cellular physiol. 233(10), 6425-6439.

9. Charach G, Rogowski O, Karniel E, et al. (2019). Monocytes may be favorable biomarker and predictor of long-term outcome in patients with chronic heart failure: a cohort study. Medicine, 98(38).

10. Bowe B, Xie $Y$, Xian $H$, et al. (2017). Association between monocyte count and risk of incident CKD and progression to ESRD. Clinical Journal of the American Society of Nephrology, 12(4), 603613.

11. Flynn MC, Pernes G, Lee MK, et al. (2019). Monocytes, macrophages, and metabolic disease in atherosclerosis. Frontiers in pharmacology, 10, 666.

12. Roy-Chowdhury E, Brauns, N, Helmke A, et al. (2021). Human CD16+ monocytes promote a proatherosclerotic endothelial cell phenotype via 
CX3CR1-CX3CL1 interaction. Cardiovascular Research, 117(6), 1510-1522.

13. Jung JH, Choi JE, Song JH, et al. (2018). Human CD36 overexpression in renal tubules accelerates the progression of renal diseases in a mouse model of folic acid-induced acute kidney injury. Kidney research and clinical practice, 37(1), 30.

14. Dounousi E, Duni A, Naka KK., et al. (2021). The innate immune system and cardiovascular disease in ESKD: monocytes and natural killer cells. Current Vascular Pharmacology, 19(1), 6376.

15. Poznyak, AV, Wu WK, Melnichenko AA, et al. (2020). Signaling pathways and key genes involved in regulation of foam cell formation in atherosclerosis. Cells, 9(3), 584.

16. Chistiakov DA, Melnichenko AA, Myasoedova VA, et al. (2017). Mechanisms of foam cell formation in atherosclerosis. Journal of molecular medicine, 95(11), 1153-1165.

17. Park SH (2021). Regulation of Macrophage Activation and Differentiation in Atherosclerosis. Journal of Lipid and Atherosclerosis, 10.

18. Yang M, and Silverstein RL (2019). CD36 signaling in vascular redox stress. Free Radical Biology and Medicine, 136, 159-171.

19. KDIGO (2012): KDIGO Clinical Practice Guideline for Anemia in chronic kidney disease, Kidney Int Suppl; 2: 283-287.

20. Carmona A, Guerrero F, Jimenez MJ, et al. (2020). Inflammation, Senescence and MicroRNAs in Chronic Kidney Disease. Frontiers in Cell and Developmental Biology, 8, 739.

21. SahBandar IN, Ndhlovu LC, Saiki K, et al. (2019). Relationship between circulating inflammatory monocytes and cardiovascular disease measures of carotid intimal thickness. Journal of atherosclerosis and thrombosis, 49791.

22. Rogacev KS, Seiler S, Zawada AM, et al. (2011). CD14++CD16+ monocytes and cardiovascular outcome in patients with chronic kidney disease. Eur Heart J.; 32(1):84-92

23. Chiu YL, Shu KH, Yang, FJ, et al. (2018). A comprehensive characterization of aggravated aging-related changes in $\mathrm{T}$ lymphocytes and monocytes in end-stage renal disease: the iESRD study. Immunity \& Ageing, 15(1), 1-10.

24. Tian K, Xu Y, Sahebkar A, et al. (2020). CD36 in atherosclerosis: pathophysiological mechanisms and therapeutic implications. Current Atherosclerosis Reports, 22(10), 1-10.

25. Choromańska $B$, Myśliwiec $P$, Choromańska $K$, et al. (2017). The role of CD36 receptor in the pathogenesis of atherosclerosis. Adv Clin Exp Med.; 26(4):717-722.

26. Glatz JC, and Luiken JF (2018). Dynamic role of the transmembrane glycoprotein CD36 (SR-B2) in cellular fatty acid uptake and utilization. Journal of lipid research, 59(7), 1084-1093.

27. Lim YJ, Sidor NA., Tonial NC, et al. (2021). Uremic Toxins in the Progression of Chronic Kidney Disease and Cardiovascular Disease: Mechanisms and Therapeutic Targets. Toxins, 13(2), 142.

28. Liakopoulos V, Jeron A, Shah A, et al. (2018). Hemodialysis-related changes in phenotypical features of monocytes. Scientific reports, 8(1), 112.

29. Girndt M, Trojanowicz B, and Ulrich, C. (2020). Monocytes in uremia. Toxins, 12(5), 340.

30.Biswas S, Gao D, Altemus J.B, et al. (2021). Circulating CD36 is increased in hyperlipidemic mice: Cellular sources and triggers of release. Free Radical Biology and Medicine, 168, 180-188. 